\section{On Improving the Performance With Bounded Continuous Feedback Laws}

Tingshu Hu and Zongli Lin

\begin{abstract}
We present controller design methods to smooth the discontinuity resulting from a piecewise linear control law which was proposed to improve the convergence performance for systems with input constraints. The continuous control laws designed in this note are explicit functions of the state and are easily implementable. We also show that the convergence performance can be further improved by using a saturated high-gain feedback law. The efficiency of the proposed methods is illustrated with the PUMA 560 robot model.
\end{abstract}

Index Terms-Constrained control, convergence rate, invariant ellipsoid, switching.

\section{INTRODUCTION}

We consider a linear system subject to input saturation and state constraint

$$
\dot{x}=A x+B u, \quad|u|_{\infty} \leq 1 ; \quad x \in \Omega_{0}
$$

where $x \in \mathbf{R}^{n}, u \in \mathbf{R}^{m},|u|_{\infty}=\max \left\{\left|u_{i}\right|, i \in[1, m]\right\}$ and $\Omega_{0}$ contains the origin in its interior. To achieve a large domain of attraction, we may try to find a large ellipsoid (see [2] and [7])

$$
\mathcal{E}(P, \rho):=\left\{x \in \mathbf{R}^{n}: x^{T} P x \leq \rho\right\} \subset \Omega_{0}
$$

with $0<P \in \mathbf{R}^{n \times n}$, such that this ellipsoid is invariant under $u=$ $F x$ and

$$
\mathcal{E}(P, \rho) \subset \mathcal{L}(F):=\left\{x \in \mathbf{R}^{n}:|F x|_{\infty} \leq 1\right\} .
$$

For simplicity, we use $\mathcal{E}(P)$ to denote $\mathcal{E}(P, 1)$. Generally, the maximization of $\mathcal{E}(P, \rho)$ would result in low-feedback gain $F$ and slow convergence rate, i.e., some eigenvalues of $A+B F$ are close to the imaginary axis. In [7], Wredenhagen and Belanger proposed a piecewise linear control (PLC) design method to reconcile large domain of attraction and good convergence performance. The basic idea is to use a linear quadratic (LQ) method to construct a sequence of nested ellipsoids

$$
\mathcal{E}\left(P_{0}, \rho_{0}\right) \supset \mathcal{E}\left(P_{1}, \rho_{1}\right) \supset \cdots \supset \mathcal{E}\left(P_{N}, \rho_{N}\right)
$$

along with corresponding feedback gain matrices $F_{i}, i=0,1, \ldots, N$, such that $\mathcal{E}\left(P_{0}, \rho_{0}\right) \subset \Omega_{0}, \mathcal{E}\left(P_{i}, \rho_{i}\right) \subset \mathcal{L}\left(F_{i}\right)$, and each $\mathcal{E}\left(P_{i}, \rho_{i}\right)$ is invariant under the feedback $u=F_{i} x, i=0,1, \ldots, N$. Also, as the index $i$ is increased, the convergence rate under the feedback $u=F_{i} x$ increases. The final controller takes the following form:

$$
u= \begin{cases}F_{N} x, & \text { if } x \in \mathcal{E}\left(P_{N}, \rho_{N}\right) \\ F_{N-1} x, & \text { if } x \in \mathcal{E}\left(P_{N-1}, \rho_{N-1}\right) \backslash \mathcal{E}\left(P_{N}, \rho_{N}\right) \\ \vdots & \\ F_{0} x, & \text { if } x \in \mathcal{E}\left(P_{0}, \rho_{0}\right) \backslash \mathcal{E}\left(P_{1}, \rho_{1}\right) .\end{cases}
$$

Manuscript received October 16, 2001. Recommended by Associate Editor A. Bemporad. This work was supported in part by the U.S. Office of Naval Research Young Investigator Program under Grant N00014-99-1-0670.

The authors are with the Department of Electrical and Computer Engineering, University of Virginia, Charlottesville, VA 22903 USA (e-mail: th7f@virginia.edu; zl5y@ virginia.edu)

Publisher Item Identifier 10.1109/TAC.2002.802739.
In this way, the domain of attraction is ensured to include the largest ellipsoid $\mathcal{E}\left(P_{0}, \rho_{0}\right)$ and as a state trajectory moves from an outer ellipsoid to an inner ellipsoid, the convergence rate is increased. Since each ellipsoid is invariant under the corresponding feedback law, the switch is safe (no chattering) and the existence and uniqueness of the solution to the closed-loop differential equation is ensured. Such a control law is referred to as PLC in [7]. Because $\mathcal{E}\left(P_{i}, \rho_{i}\right) \subset \mathcal{L}\left(F_{i}\right)$, $i=0,1, \ldots, N$, the control $u$ will never exceed the saturation bound if the initial state $x_{0} \in \mathcal{E}\left(P_{0}, \rho_{0}\right)$.

Since $F_{i}$ is generally different from $F_{i-1}$, the control $u$ in (2) is discontinuous at the switching surface $\partial \mathcal{E}\left(P_{i}, \rho_{i}\right)$, the boundary of the ellipsoid $\mathcal{E}\left(P_{i}, \rho_{i}\right)$. Effort has been made to smoothen the discontinuity in [3]-[6], etc. In [6], a continuous feedback law was constructed from the linear combination of $F_{k}$ and $F_{k+1}$. Since this simple interpolation may cause the control to exceed the constraint, smaller bounds on the control were imposed and the ellipsoids were required to be "tightly" nested ( $P_{k}-P_{k+1}$ should be sufficiently small). By using the gain scheduling methods in [3]-[5], it has been shown that the discontinuity can be smoothened by using a continuum of ellipsoids $\mathcal{E}(P(\varepsilon))$, where $\varepsilon$ is a scheduling variable. The essence of these gain scheduling methods is the following. For every $\varepsilon>0, P(\varepsilon)$ is solved from a parameter dependent Riccati equation along with an LQ gain matrix $F(\varepsilon)$. As $\varepsilon$ is increased, the ellipsoid $\mathcal{E}(P(\varepsilon))$ becomes smaller and the convergence rate within $\mathcal{E}(P(\varepsilon))$ is increased. The gain scheduling idea is to associate each $x \in \mathbf{R}^{n}$ with a parameter $\varepsilon$, or to define a function $\varepsilon(x): x \mapsto \varepsilon$. The final controller has the form of $u=F(\varepsilon(x)) x$. Since the function $\varepsilon(x)$ is generally very hard to compute, technical issues are involved in controller implementation. These issues were considered in [3] and a method to simplify the computation of $\varepsilon(x)$ was proposed. The proposed method involves solving a convex optimization problem for every point $x$ in the state space.

This note is intended to propose explicit controller structures which would achieve the objective of improving the convergence performance using continuous control laws. For easy reference, here we collect some simple mathematical facts as follows.

Fact 1: For two ellipsoids $\mathcal{E}\left(P_{1}\right)$ and $\mathcal{E}\left(P_{2}\right)$

$$
\begin{aligned}
\mathcal{E}\left(P_{1}\right) \subset \mathcal{E}\left(P_{2}\right) & \Longleftrightarrow P_{1} \geq P_{2} \\
\mathcal{E}\left(P_{1}\right) \subset \operatorname{int}\left(\mathcal{E}\left(P_{2}\right)\right) & \Longleftrightarrow P_{1}>P_{2}
\end{aligned}
$$

where $\operatorname{int}\left(\mathcal{E}\left(P_{2}\right)\right)=\left\{x \in \mathbf{R}^{n}: x^{T} P_{2} x<1\right\}$ is the interior of $\mathcal{E}\left(P_{2}\right)$. For an ellipsoid $\mathcal{E}(P)$ and a matrix $F \in \mathbf{R}^{m \times n}$

$$
\begin{aligned}
\mathcal{E}(P) \subset \mathcal{L}(F) & \Longleftrightarrow f_{i}^{T} f_{i} \leq P, \quad i \in[1, m] \\
& \Longleftrightarrow f_{i} P^{-1} f_{i}^{T} \leq 1, \quad i \in[1, m] \\
& \Longleftrightarrow\left[\begin{array}{cc}
1 & f_{i} P^{-1} \\
P^{-1} f_{i}^{T} & P^{-1}
\end{array}\right] \geq 0, \quad \in[1, m] .
\end{aligned}
$$

\section{A CONTINUOUS FEEDBACK LAW FOR IMPROVING THE PERFORMANCE}

We consider the system

$$
\dot{x}=A x+B u, \quad|u|_{\infty} \leq 1 ; \quad x \in \Omega_{0}
$$

with a two stage switching feedback law

$$
u= \begin{cases}F_{1} x, & \text { if } x \in \mathcal{E}\left(P_{1}\right) \\ F_{0} x, & \text { if } x \in \mathcal{E}\left(P_{0}\right) \backslash \mathcal{E}\left(P_{1}\right)\end{cases}
$$


where

$$
\begin{aligned}
& P_{0}<P_{1} \quad \Omega_{0} \supset \mathcal{E}\left(P_{0}\right) \\
& \mathcal{E}\left(P_{0}\right) \subset \mathcal{L}\left(F_{0}\right) \quad \mathcal{E}\left(P_{1}\right) \subset \mathcal{L}\left(F_{1}\right) \\
& \left(A+B F_{0}\right)^{T} P_{0}+P_{0}\left(A+B F_{0}\right) \leq-\alpha_{0} P_{0} \\
& \left(A+B F_{1}\right)^{T} P_{1}+P_{1}\left(A+B F_{1}\right) \leq-\alpha_{1} P_{1}
\end{aligned}
$$

and $0<\alpha_{0}<\alpha_{1}$. Assume that $\alpha_{0}$ and $\alpha_{1}$ are the maximal positive numbers that satisfy (6) and (7), respectively. The inequality $\alpha_{0}<\alpha_{1}$ implies that the convergence rate of the Lyapunov function $V_{1}(x)=$ $x^{T} P_{1} x$ under $u=F_{1} x$ is greater than that of $V_{0}(x)=x^{T} P_{0} x$ under $u=F_{0} x$. We consider a feedback law (4) of only one switch because the method to be proposed can be readily extended to smoothen the discontinuity of a controller with multiple switches. Actually, because the proposed continuous feedback law guarantees a progressively increasing convergence rate, we only need to use the outmost and the innermost ellipsoids $\mathcal{E}\left(P_{0}, \rho_{0}\right)$ and $\mathcal{E}\left(P_{N}, \rho_{N}\right)$ along with their corresponding feedback gain matrices $F_{0}$ and $F_{N}$. Without loss of generality, we have assumed that $\rho_{0}=\rho_{1}=1$. Otherwise, $\rho_{0}$ and $\rho_{1}$ can be absorbed into the matrices $P_{0}$ and $P_{1}$.

The control (4) is discontinuous at the surface of the inner ellipsoid, $\partial \mathcal{E}\left(P_{1}\right)$. The main idea for smoothening this discontinuity is to construct a continuum of ellipsoids $\mathcal{E}(P(\gamma)), \gamma \in[0,1]$, between $\mathcal{E}\left(P_{0}\right)$ and $\mathcal{E}\left(P_{1}\right)$, progressively shrinking, along with a continuum of feedback matrices $F(\gamma)$, such that

$$
\mathcal{E}(P(\gamma)) \subset \mathcal{L}(F(\gamma))
$$

and

$$
(A+B F(\gamma))^{T} P(\gamma)+P(\gamma)(A+B F(\gamma)) \leq-\alpha(\gamma) P(\gamma)
$$

with $\alpha(\gamma)$ monotonically increasing as $\gamma$ changes from zero to one. For $x \in \partial \mathcal{E}(P(\gamma))$, we use the control $u=F(\gamma) x$. Suppose that for every $x \in \mathcal{E}\left(P_{0}\right) \backslash \operatorname{int}\left(\mathcal{E}\left(P_{1}\right)\right)$, there exists a unique $\gamma \in[0,1]$ such that $x^{T} P(\gamma) x=1$, we can then define

$$
\gamma(x):=\left\{\gamma \in[0,1]: x^{T} P(\gamma) x=1\right\}
$$

and the feedback law can be simply written as

$$
u=F(\gamma(x)) x .
$$

The control law (9) is implementable if the function $\gamma(x)$ and the feedback matrix $F(\gamma(x))$ can be computed efficiently on line. That is, we should be able to tell which ellipsoid surface the state $x$ is on. This depends on how we design the functions $P(\gamma)$ and $F(\gamma)$.

The following are the functions we propose. Let

$$
\begin{aligned}
& Q_{0}=P_{0}^{-1} \\
& Q_{1}=P_{1}^{-1} \\
& H_{0}=F_{0} Q_{0} \\
& H_{1}=F_{1} Q_{1} .
\end{aligned}
$$

Define

$$
\begin{aligned}
Q(\gamma) & :=(1-\gamma) Q_{0}+\gamma Q_{1} \\
H(\gamma) & :=(1-\gamma) H_{0}+\gamma H_{1}
\end{aligned}
$$

and

$$
\begin{aligned}
& P(\gamma):=Q(\gamma)^{-1} \\
& F(\gamma):=H(\gamma) P(\gamma) .
\end{aligned}
$$

It is clear that $Q(\gamma)>0$ for all $\gamma \in[0,1]$. Hence, $Q(\gamma), H(\gamma), P(\gamma)$ and $F(\gamma)$ are all continuous in $\gamma$ over the interval $[0,1]$. The same function $Q(\gamma)$ was used in [3], where $F(\gamma)$ was the solution to a Riccati equation. In what follows, we show that, with $F(\gamma)$ defined as previously shown, the continuous feedback law $u=F(\gamma(x)) x$ possesses all the desired properties. We will also provide an explicit formula to compute $\gamma(x)$.

Theorem 1: With $P(\gamma)$ defined in (11), there exists a unique $\gamma \in$ $[0,1]$ such that $x^{T} P(\gamma) x=1$ for every $x \in \mathcal{E}\left(P_{0}\right) \backslash \operatorname{int}\left(\mathcal{E}\left(P_{1}\right)\right)$. With $\gamma(x)$ defined in (8), we have

$$
\gamma(x)=\lambda_{\min }\left[\left(Q_{0}-Q_{1}\right)^{-(1 / 2)}\left(Q_{0}-x x^{T}\right)\left(Q_{0}-Q_{1}\right)^{-(1 / 2)}\right]
$$

for $x \in \mathcal{E}\left(P_{0}\right) \backslash \operatorname{int}\left(\mathcal{E}\left(P_{1}\right)\right)$. Let $\gamma(x)=1$ for $x \in \operatorname{int}\left(\mathcal{E}\left(P_{1}\right)\right)$. Then, the control $u=F(\gamma(x)) x$ is continuous in $x$ and $|F(\gamma(x)) x| \leq 1$ for all $x \in \mathcal{E}\left(P_{0}\right)$. Moreover, each ellipsoid $\mathcal{E}(P(\gamma)), \gamma \in[0,1]$ is invariant and every trajectory starting from $x_{0} \in \mathcal{E}\left(P_{0}\right)$ will converge to the origin with increasing rate.

Before proving Theorem 1, we present two lemmas. Define

$$
\begin{aligned}
\alpha(\gamma):=\max \left\{\alpha>0:(A+B F(\gamma))^{T} P(\gamma)\right. & \\
& +P(\gamma)(A+B F(\gamma)) \leq-\alpha P(\gamma)\} .
\end{aligned}
$$

Then, $\alpha(\gamma)$ is the convergence rate of $V(x)=x^{T} P(\gamma) x$ under the linear control $u=F(\gamma) x$.

\section{Lemma 1:}

1) $\mathcal{E}(p(\gamma))$ shrinks as $\gamma$ increases, namely, if $\gamma_{1}<\gamma_{2}$, then $\mathcal{E}\left(P\left(\gamma_{2}\right)\right) \subset \operatorname{int}\left(\mathcal{E}\left(P\left(\gamma_{1}\right)\right)\right)$.

2) For all $\gamma \in[0,1], \mathcal{E}(P(\gamma)) \subset \mathcal{L}(F(\gamma))$.

3) $\alpha(\gamma)$ is strictly increasing for $\gamma \in[0,1]$.

Proof: From (5), we have $Q_{0}-Q_{1}=P_{0}^{-1}-P_{1}^{-1}>0$.

1) If $\gamma_{1}<\gamma_{2}$, then

$$
Q\left(\gamma_{1}\right)-Q\left(\gamma_{2}\right)=\left(\gamma_{2}-\gamma_{1}\right)\left(Q_{0}-Q_{1}\right)>0 .
$$

Hence, $P\left(\gamma_{1}\right)<P\left(\gamma_{2}\right)$ and it follows from Fact 1 that $\mathcal{E}\left(P\left(\gamma_{2}\right)\right) \subset \operatorname{int}\left(\mathcal{E}\left(P\left(\gamma_{1}\right)\right)\right.$.

2) Since $\mathcal{E}\left(P_{0}\right) \subset \mathcal{L}\left(F_{0}\right)$ and $\mathcal{E}\left(P_{1}\right) \subset \mathcal{L}\left(F_{1}\right)$, it follows from Fact 1 that

$$
\begin{aligned}
& {\left[\begin{array}{cc}
1 & f_{0 i} P_{0}^{-1} \\
P_{0}^{-1} f_{0 i}^{T} & P_{0}^{-1}
\end{array}\right] \geq 0} \\
& {\left[\begin{array}{cc}
1 & f_{1 i} P_{1}^{-1} \\
P_{1}^{-1} f_{1 i}^{T} & P_{1}^{-1}
\end{array}\right] \geq 0, \quad i \in[1, m]}
\end{aligned}
$$

i.e.,

$$
\left[\begin{array}{cc}
1 & h_{0 i} \\
h_{0 i}^{T} & Q_{0}
\end{array}\right] \geq 0, \quad\left[\begin{array}{cc}
1 & h_{1 i} \\
h_{1 i}^{T} & Q_{1}
\end{array}\right] \geq 0, \quad i \in[1, m] .
$$

By convexity, we have

$$
\left[\begin{array}{cc}
1 & h_{i}(\gamma) \\
h_{i}^{T}(\gamma) & Q(\gamma)
\end{array}\right] \geq 0, \quad i \in[1, m], \quad \gamma \in[0,1]
$$

That is

$$
\begin{aligned}
& {\left[\begin{array}{cc}
1 & f_{i}(\gamma) P^{-1}(\gamma) \\
P^{-1}(\gamma) f_{i}^{T}(\gamma) & P^{-1}(\gamma)
\end{array}\right] \geq 0} \\
& i \in[1, m], \quad \gamma \in[0,1] .
\end{aligned}
$$

Therefore, by Fact 1, we have $\mathcal{E}(P(\gamma)) \subset \mathcal{L}(F(\gamma))$ for all $\gamma \in$ $[0,1]$.

3) By multiplying both sides of (6) and (7) with $Q_{0}$ and $Q_{1}$, respectively, we obtain

$$
\begin{aligned}
& Q_{0} A^{T}+A Q_{0}+H_{0}^{T} B^{T}+B H_{0} \leq-\alpha_{0} Q_{0} \\
& Q_{1} A^{T}+A Q_{1}+H_{1}^{T} B^{T}+B H_{1} \leq-\alpha_{1} Q_{1} .
\end{aligned}
$$

By convexity, we have

$$
\begin{aligned}
& Q(\gamma) A^{T}+A Q(\gamma)+H(\gamma)^{T} B^{T}+B H(\gamma) \\
& \quad \leq-(1-\gamma) \alpha_{0} Q_{0}-\gamma \alpha_{1} Q_{1}, \quad \gamma \in[0,1] .
\end{aligned}
$$

Since $\alpha_{1}>\alpha_{0}$ and $Q_{1}>0$, we see that

$(1-\gamma) \alpha_{0} Q_{0}+\gamma \alpha_{1} Q_{1}=\alpha_{0} Q(\gamma)+\gamma\left(\alpha_{1}-\alpha_{0}\right) Q_{1}>\alpha_{0} Q(\gamma)$. 
It follows that $\alpha(\gamma)>\alpha_{0}$ for all $\gamma \in(0,1]$. To show that $\alpha\left(\gamma_{2}\right)>\alpha\left(\gamma_{1}\right)$ for $\gamma_{2}>\gamma_{1}$, we observe that

$$
\begin{aligned}
\gamma_{2} & =\left(1-\frac{\gamma_{2}-\gamma_{1}}{1-\gamma_{1}}\right) \gamma_{1}+\frac{\gamma_{2}-\gamma_{1}}{1-\gamma_{1}} \\
Q\left(\gamma_{2}\right) & =\left(1-\frac{\gamma_{2}-\gamma_{1}}{1-\gamma_{1}}\right) Q\left(\gamma_{1}\right)+\frac{\gamma_{2}-\gamma_{1}}{1-\gamma_{1}} Q_{1}
\end{aligned}
$$

and

$$
H\left(\gamma_{2}\right)=\left(1-\frac{\gamma_{2}-\gamma_{1}}{1-\gamma_{1}}\right) H\left(\gamma_{1}\right)+\frac{\gamma_{2}-\gamma_{1}}{1-\gamma_{1}} H_{1} .
$$

Following the same procedure by replacing $Q_{0}$ with $Q\left(\gamma_{1}\right), H_{0}$ with $H\left(\gamma_{1}\right)$, and $\gamma$ with $\left(\gamma_{2}-\gamma_{1}\right) /\left(1-\gamma_{1}\right)$, we can show that $\alpha\left(\gamma_{2}\right)>\alpha\left(\gamma_{1}\right)$ for $\gamma_{2}>\gamma_{1}$.

Lemma 2: For every $x \in \mathcal{E}\left(P_{0}\right) \backslash \operatorname{int}\left(\mathcal{E}\left(P_{1}\right)\right)$, there exists a unique $\gamma \in[0,1]$ such that $x^{T} P(\gamma) x=1$. Let $\gamma(x)$ be defined in (8). Then, for $x \in \mathcal{E}\left(P_{0}\right) \backslash \operatorname{int}\left(\mathcal{E}\left(P_{1}\right)\right)$

$$
\gamma(x)=\lambda_{\min }\left[\left(Q_{0}-Q_{1}\right)^{-(1 / 2)}\left(Q_{0}-x x^{T}\right)\left(Q_{0}-Q_{1}\right)^{-(1 / 2)}\right] .
$$

Proof: For each $x \in \mathcal{E}\left(P_{0}\right) \backslash \operatorname{int}\left(\mathcal{E}\left(P_{1}\right)\right)$, we have $x^{T} P_{0} x \leq 1$ and $x^{T} P_{1} x \geq 1$, i.e., $x^{T} P(0) x \leq 1$ and $x^{T} P(1) x \geq 1$. Since $P(\gamma)$ is continuous in $\gamma$ for $\gamma \in[0,1]$, there exists a $\gamma \in[0,1]$ such that $x^{T} P(\gamma) x=1$. By Lemma 1 (item 1), there exists a unique $\gamma \in[0,1]$ such that $x^{T} P(\gamma) x=1$. Hence, the function $\gamma(x)$ is well defined by (8).

Let $\gamma$ be the unique number in $[0,1]$ such that $x^{T} P(\gamma) x=1$, i.e., $x \in \partial \mathcal{E}(P(\gamma))$. By Lemma 1 (item 1), $x \in \operatorname{int}\left(\mathcal{E}\left(P\left(\gamma_{1}\right)\right)\right)$ for all $\gamma_{1} \in[0, \gamma)$, i.e.,

$$
x^{T} P\left(\gamma_{1}\right) x<1 \quad \forall \gamma_{1} \in[0, \gamma) .
$$

It follows from the Schur complement that

$$
\begin{aligned}
& {\left[\begin{array}{cc}
1 & x^{T} \\
x & Q\left(\gamma_{1}\right)
\end{array}\right]=\left[\begin{array}{cc}
1 & x^{T} \\
x & Q_{0}-\gamma_{1}\left(Q_{0}-Q_{1}\right)
\end{array}\right]>0 } \\
\Longleftrightarrow & Q_{0}-\gamma_{1}\left(Q_{0}-Q_{1}\right)-x x^{T}>0 \\
\Longleftrightarrow & \gamma_{1} I<\left(Q_{0}-Q_{1}\right)^{-(1 / 2)}\left(Q_{0}-x x^{T}\right)\left(Q_{0}-Q_{1}\right)^{-(1 / 2)} \\
& \gamma_{1} \in[0, \gamma) .
\end{aligned}
$$

By continuity, we have

$$
\gamma I \leq\left(Q_{0}-Q_{1}\right)^{-(1 / 2)}\left(Q_{0}-x x^{T}\right)\left(Q_{0}-Q_{1}\right)^{-(1 / 2)} .
$$

From $x^{T} P(\gamma) x=1$, we have

$$
\begin{aligned}
0 & =\operatorname{det}\left[\begin{array}{cc}
1 & x^{T} \\
x & Q(\gamma)
\end{array}\right] \\
& =\operatorname{det}\left[Q(\gamma)-x x^{T}\right] \\
& =\operatorname{det}\left[Q_{0}-\gamma\left(Q_{0}-Q_{1}\right)-x x^{T}\right] .
\end{aligned}
$$

Hence

$$
\begin{aligned}
\operatorname{det}\left[\gamma I-\left(Q_{0}-Q_{1}\right)^{-(1 / 2)}\left(Q_{0}-\right.\right. & \left.x x^{T}\right) \\
& \left.\times\left(Q_{0}-Q_{1}\right)^{-(1 / 2)}\right]=0
\end{aligned}
$$

which implies that $\gamma$ is an eigenvalue of the matrix $\left(Q_{0}-\right.$ $\left.Q_{1}\right)^{-(1 / 2)}\left(Q_{0}-x x^{T}\right)\left(Q_{0}-Q_{1}\right)^{-(1 / 2)}$. In view of (14), we obtain (13).

Proof of Theorem 1: The first statement and (12) have been proved in Lemma 2. From the continuity of the eigenvalues of a matrix in its elements, it follows that $\gamma(x)$ is continuous in $x$ at every $x \in \mathcal{E}\left(P_{0}\right) \backslash \operatorname{int}\left(\mathcal{E}\left(P_{1}\right)\right)$. Since $\gamma(x)=1$ for all $x \in \partial \mathcal{E}\left(P_{1}\right)$, the function $\gamma(x)$ can be extended continuously to all $x \in \mathcal{E}\left(P_{0}\right)$ by letting $\gamma(x)=1$ for $x \in \mathcal{E}\left(P_{1}\right)$. Since $F(\gamma)$ is continuous in $\gamma$, the control $u=F(\gamma(x)) x$ is continuous in $x$. The claim that $|F(\gamma(x)) x|_{\infty} \leq 1$ for all $x \in \mathcal{E}\left(P_{0}\right)$ follows directly from Lemma 1 (item 2).
Now, we consider the convergence of trajectories. Under the control $u=F(\gamma(x)) x$, on the boundary of each $\mathcal{E}(P(\gamma)), \gamma \in[0,1]$

$$
\begin{aligned}
x^{T} P(\gamma) \dot{x} & =\frac{1}{2} x^{T}\left((A+B F(\gamma))^{T} P+P(A+B F(\gamma)) x\right. \\
& \leq-\frac{1}{2} \alpha(\gamma) x^{T} P(\gamma) x<0 .
\end{aligned}
$$

We also have $x^{T} P_{1} \dot{x}<0$ for all $x \in \mathcal{E}\left(P_{1}\right)$. Hence, all the trajectories starting from the boundary of $\mathcal{E}(P(\gamma))$ will be in the interior of $\mathcal{E}(P(\gamma))$ for all $t>0$. This proves the invariance of $\mathcal{E}(P(\gamma))$. Moreover, the convergence rate $\alpha(\gamma)$ increases as the trajectory enters the inner ellipsoids. Therefore, if a trajectory strats from $x_{0} \in \mathcal{E}\left(P_{0}\right)$, it will converge to the origin.

\section{Further IMPROVEMENT OF THE CONVERGENCE PeRformance}

As can be seen from Lemma 1 (item 2), the control law constructed in Section II satisfies the control constraint by avoiding saturation. Since $\mathcal{E}(P(\gamma)) \subset \mathcal{L}(F(\gamma))$, there are at most two intersections between the ellipsoid $\mathcal{E}(P(\gamma))$ and a pair of hyperplanes, $f_{i}(\gamma) x= \pm 1$. Hence, the control $u_{i}=f_{i}(\gamma) x$ may take the maximal value \pm 1 only at two points on $\partial \mathcal{E}(P(\gamma))$. Along a trajectory, the control signal could be well below the saturation level most of the time. This means that the capacity of the actuators is not fully utilized and we still have much potential to improve the convergence performance. In [1], it is shown that the control law that maximizes the convergence rate of a Lyapunov function $V(x)=x^{T} P x$ under actuator saturation is simply $u_{i}=-\operatorname{sign}\left(b_{i}^{T} P x\right), i=1,2, \ldots, m$. Due to the discontinuity of this bang-bang control, a saturated high gain linear feedback law of the form $u=-\operatorname{sat}\left(k B^{T} P x\right)$ is proposed to achieve a suboptimal convergence rate. Here, sat $(\cdot)$ is the standard vector-valued saturation function: $\{\operatorname{sat}(u)\}_{i}=\operatorname{sign}\left(u_{i}\right) \min \left\{\left|u_{i}\right|, 1\right\}$. It is also shown that the maximal convergence rate depends on the choice of the $P$ matrix (see [1, Ch. 11] for more details). Generally, the objective of producing a high convergence rate conflicts with the objective of achieving a large invariant ellipsoid. In other words, if $P$ is chosen such that the maximal convergence rate is high, then the largest ellipsoid $\mathcal{E}(P, \rho)$ that can be made invariant would be small. A simple way to reconcile the objective of producing a large invariant ellipsoid and that of achieving a high convergence rate with a control $u=-\operatorname{sat}\left(k B^{T} P x\right)$ is also to adjust the $P$ matrix according to the size of the state. Using the method in Section II, a state dependent matrix $P(\gamma(x))$ can be determined. While the state feedback $u=F(\gamma(x)) x$ increases the convergence rate as the trajectory enters smaller ellipsoid $\mathcal{E}(P(\gamma))$, a state feedback of the form $u=-\operatorname{sat}\left(k B^{T} P(\gamma(x)) x\right)$ with a high gain $k$ can be used for further improvement of the convergence performance.

Theorem 2: Let $F_{0}=-G_{0} B^{T} P_{0}$ and $F_{1}=-G_{1} B^{T} P_{1}$ be the feedback matrices of two LQ controllers such that $\mathcal{E}\left(P_{0}\right) \subset \mathcal{L}\left(F_{0}\right), \mathcal{E}\left(P_{1}\right) \subset \mathcal{L}\left(F_{1}\right)$ and $P_{1}<P_{0}$. Assume that $G_{0}=\operatorname{diag}\left\{g_{01}, g_{02}, g_{03}\right\}>0$, and $G_{1}=\operatorname{diag}\left\{g_{11}, g_{12}, g_{13}\right\}>0$. Let $k_{0}=\max \left\{g_{01}, g_{02}, g_{03}, g_{11}, g_{12}, g_{13}\right\}$. Then, for any $k>k_{0}$, under the control of

$$
u=-\operatorname{sat}\left(k B^{T} P(\gamma(x)) x\right)
$$

all the trajectories starting from $\mathcal{E}\left(P_{0}\right)$ will converge to the origin. The convergence rate increases as $k$ increases.

Proof: Denote $G(\gamma)=(1-\gamma) G_{0}+\gamma G_{1}$. Then, $0<G(\gamma) \leq$ $k_{0} I$ for all $\gamma \in[0,1]$ and $F(\gamma)=-G(\gamma) B^{T} P(\gamma)$. It follows from Theorem 1 that under the control of $u=F(\gamma(x)) x$, all the trajectories starting from $\mathcal{E}\left(P_{0}\right)$ will converge to the origin. We also have $|F(\gamma(x)) x|_{\infty} \leq 1$ for all $x \in \mathcal{E}\left(P_{0}\right)$. The fact that the convergence rate is faster under (16) for $k \geq k_{0}$ can be seen from

$$
\begin{aligned}
-x^{T} P(\gamma) b_{i} \text { sat }\left(k b_{i}^{T} P(\gamma) x\right) & \leq-x^{T} P(\gamma) b_{i} \text { sat }\left(g_{i}(\gamma) b_{i}^{T} P(\gamma) x\right) \\
& =x^{T} P(\gamma) b_{i} f_{i}(\gamma) x
\end{aligned}
$$



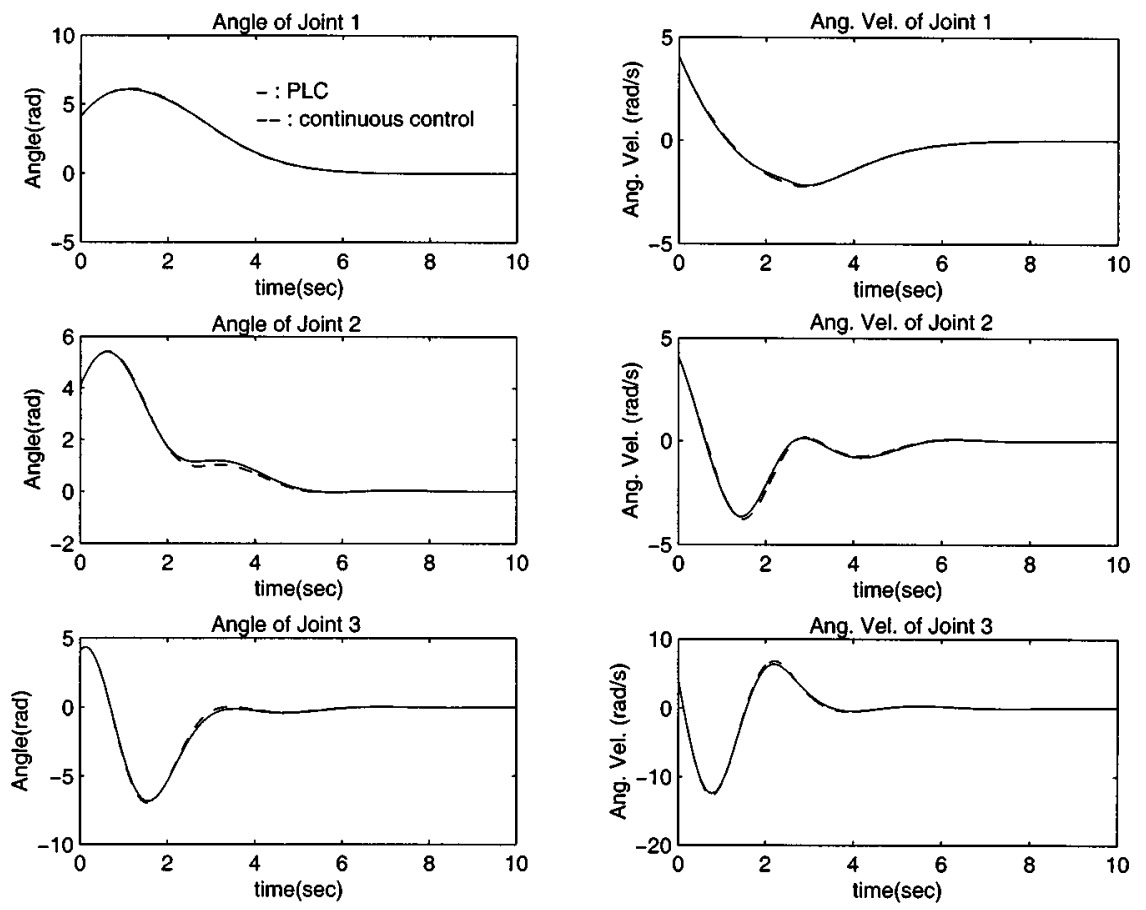

Fig. 1. Time responses of the states: PLC versus continuous control.

where $g_{i}(\gamma)$ is the $i$ th diagonal element of $G(\gamma)$. As $k$ increases, the left-hand side of (17) decreases. Hence, the convergence rate increases as $k$ increases.

Since both sat $(\cdot)$ and $P(\gamma(\cdot))$ are continuous functions, the control (16) is continuous in $x$. We call (16) the fast continuous control law. As will be seen in the example, the feedback law (16) can improve the convergence rate significantly over $u=F(\gamma(x)) x$.

Example: We consider the Puma 560 robot model that was used in [7]. The robot has three joints: the trunk (Joint 1), the shoulder (Joint 2 ), and the elbow (Joint 3). These joints are controlled by three actuators which are subject to different saturation bounds, $97.8 \mathrm{Nm}, 136.4$ $\mathrm{Nm}$, and $89.4 \mathrm{Nm}$, respectively. The linear model was calculated about $\left(\theta_{1}, \theta_{2}, \theta_{3}\right)=\left(57^{\circ}, 115^{\circ}, 172^{\circ}\right)$ with resulting system matrices

$$
\begin{aligned}
A & =\left[\begin{array}{cccccc}
0 & 0 & 0 & 1 & 0 & 0 \\
0 & 0 & 0 & 0 & 1 & 0 \\
0 & 0 & 0 & 0 & 0 & 1 \\
0 & -0.0451 & -0.0451 & 0 & 0 & 0 \\
0 & -0.0457 & -0.0457 & 0 & 0 & 0 \\
0 & -4.5551 & -4.5551 & 0 & 0 & 0
\end{array}\right] \\
B & =\left[\begin{array}{cccc}
0 & 0 & 0 & 0 \\
0 & 0 & 0 \\
0 & 0 & 0.0026 \\
0.0925 & 0.0000 & 0.0952 \\
0.0000 & 0.0979 & -0.3616 \\
0.0026 & -0.0952 & 0.36
\end{array}\right] .
\end{aligned}
$$

In [7], a PLC control law with five switches was designed. Based on the outmost and the innermost ellipsoids of [7], with corresponding feedback matrices, we designed a continuous feedback law of the form $u=F(\gamma(x)) x$. (Here, we need to take into account the nonunity saturation bounds of the three actuators). Figs. 1 and 2 illustrate the simulation results under the PLC law and the continuous feedback law. The initial condition is $x_{0}=10\left[\begin{array}{llllll}1 & 1 & 1 & 1 & 1 & 1\end{array}\right]^{T} / \sqrt{6}$, which is the
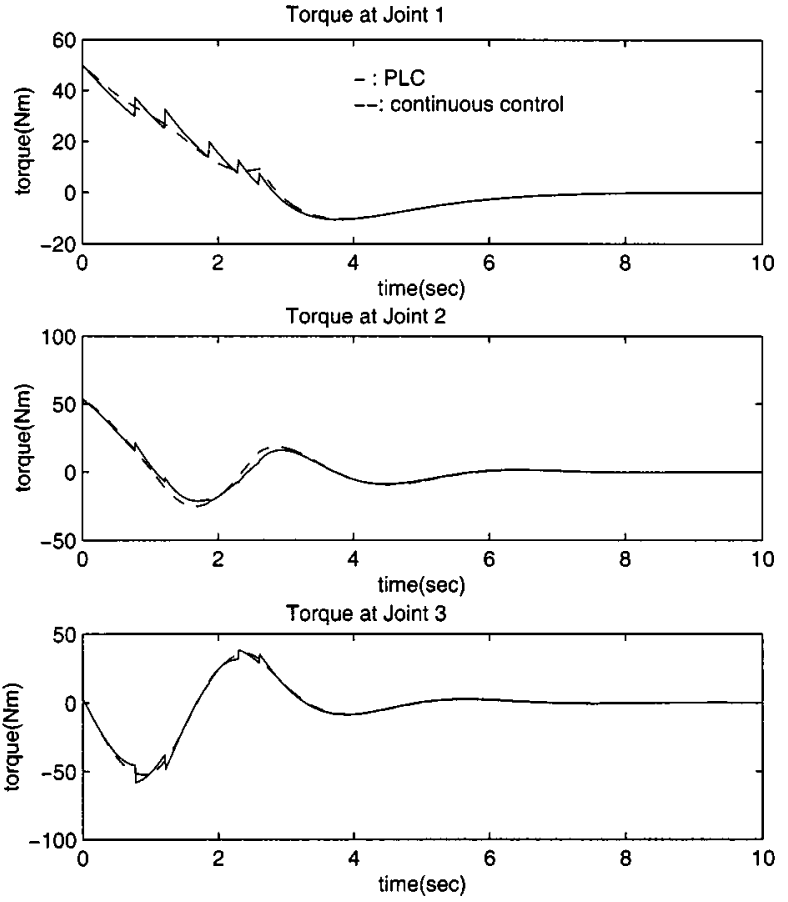

Fig. 2. Control signals: PLC versus continuous control.

same as that in [7]. From Fig. 1, we can see that the time response of the states under the PLC control and that under the continuous feedback control are almost identical. The control signals under the PLC law are however discontinuous and display big spikes (see Fig. 2). These spikes can be reduced by increasing the number of the nested ellipsoids and the controllers used in switching. However, this would increase the numerical burden when determining the smallest ellipsoid that includes a given state and would also increase the data storage for the controller. 

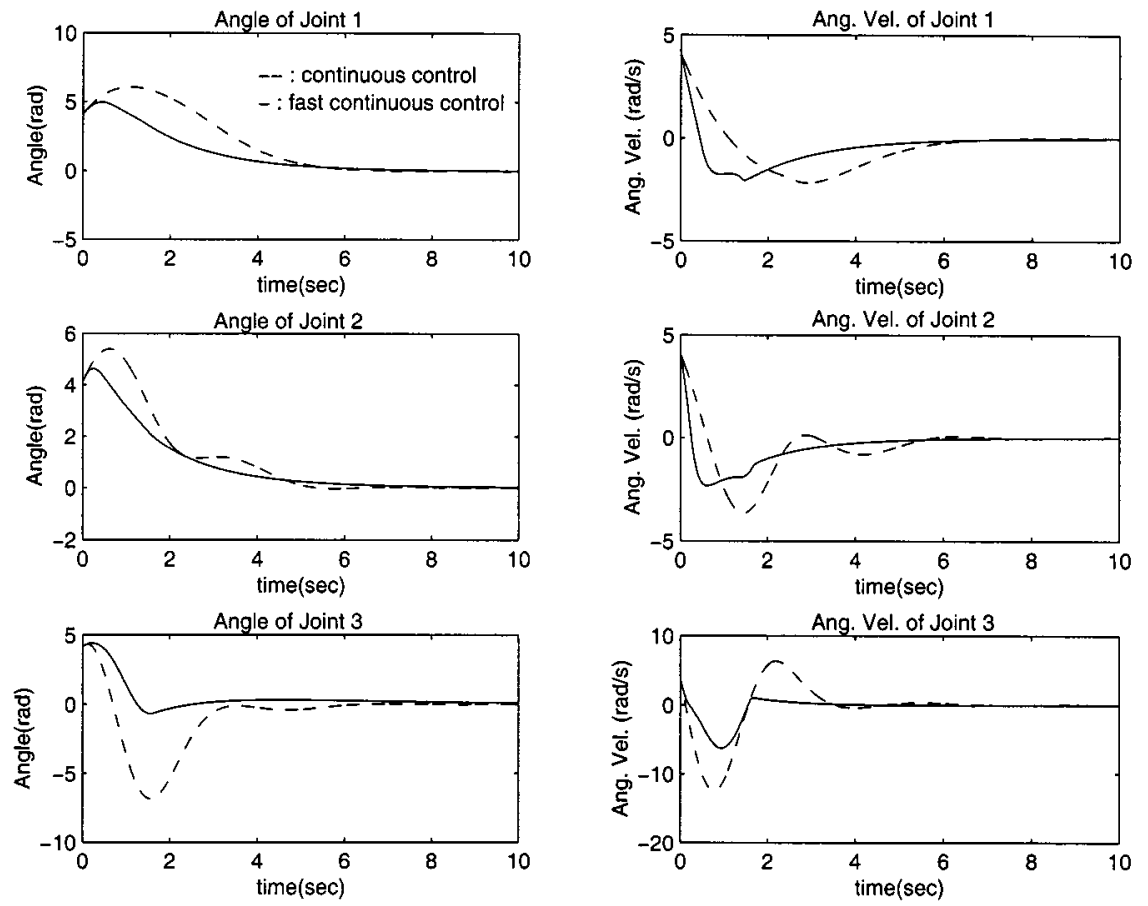

Fig. 3. Time response of the states: continuous control versus fast continuous control.
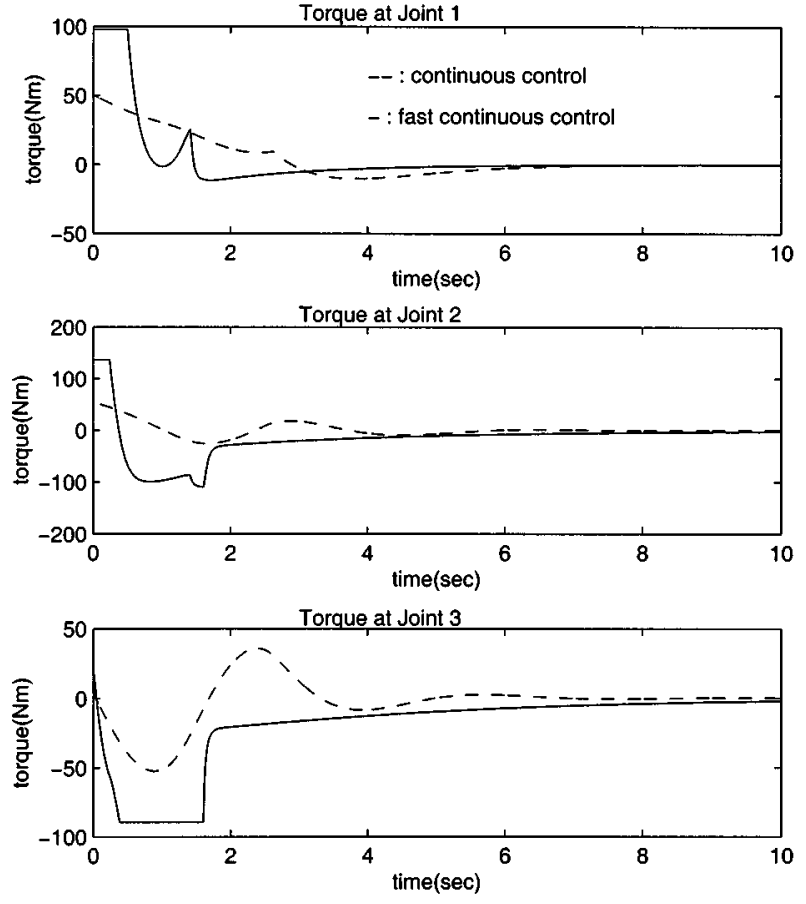

Fig. 4. The control signals: continuous control versus fast continuous control.

The control signals under the continuous control law are continuous, as expected.

From Fig. 2, we also see that the control signals are well below the saturation level. This indicates that there is a potential for further improvement of the performance. We use the controller (16) for this purpose. Recall that we have assumed unity saturation level in (1) and (16) is only suitable for systems with unity saturation level. To transform the system into the standard form of (1), let $\Lambda=\operatorname{diag}\{97.8,136.4,89.4\}, \bar{B}=B \Lambda$ and $\bar{u}=\Lambda^{-1} u$. Then the system $\dot{x}=A x+\bar{B} \bar{u}$ has a unity saturation level. For this system, the fast continuous control law is $\bar{u}=-\operatorname{sat}\left(k \bar{B}^{T} P(\gamma(x)) x\right.$. Equivalently, $u=\Lambda \bar{u}=-\Lambda \operatorname{sat}\left(k \Lambda B^{T} P(\gamma(x)) x\right)$. The actual controllers have the following forms:

$$
\left\{\begin{array}{l}
u_{1}=-97.8 \text { sat }\left(97.8 k b_{1}^{T} P(\gamma(x)) x\right) \\
u_{2}=-136.4 \text { sat }\left(136.4 k b_{2}^{T} P(\gamma(x)) x\right) \\
u_{3}=-89.4 \text { sat }\left(89.4 k b_{3}^{T} P(\gamma(x)) x\right) .
\end{array}\right.
$$

Here, in the construction of $P(\gamma)$, we have used the outmost and the innermost ellipsoids in [7]. To make full use of the actuator capacities, we have taken $k=6$ in simulation. Figs. 3 and 4 illustrate the simulation results under the control (18) (the solid curves) as compared with those under the control $u=F(\gamma(x)) x$ (the dashed curves). From Fig. 3, we see that the performance of the state response is significantly improved by using the fast continuous control law (18). Fig. 4 shows that this control law has utilized more potential of the actuator capacities. We notice that there is a sharp turn (not discontinuity) in the torque at Joint 1 . This may happen when the state trajectory enters the smaller ellipsoid $\mathcal{E}\left(P_{1}\right)$, since at the intersection between a trajectory with the boundary of this ellipsoid, the function $\gamma(x)$ is continuous but not differentiable in $x$.

\section{CONCLUSION}

We developed simple continuous feedback laws for improving the convergence performance of linear systems subject to actuator and state constraints. The control laws are expressed as explicit functions of the state and are easily implementable. The efficiency of the proposed methods is illustrated with a PUMA 560 robot model.

\section{REFERENCES}

[1] T. Hu and Z. Lin, Control Systems With Actuator Saturation: Analysis and Design. Boston, MA: Birkhäuser, 2001.

[2] — , "On enlarging the basin of attraction for linear systems under saturated linear feedback," Syst. Control Lett., vol. 40, no. 1, pp. 59-69, May 2000.

[3] A. Megretski, " $L_{2}$ BIBO output feedback stabilization with saturated control," in Proc. 13th IFAC World Congr., vol. D, 1996, pp. 435-440. 
[4] R. Suarez, J. Alvarez-Ramirez, and J. Solis-Daun, "Linear systems with bounded inputs: Global stabilization with eigenvalue placement," Int. J. Robust Nonlinear Control, vol. 7, pp. 835-845, 1997.

[5] A. R. Teel, "Linear systems with input nonlinearities: Global stabilization by scheduling a family of $H_{\infty}$-type controllers," Int. J. Robust Nonlinear Control, vol. 5, pp. 399-441, 1995.

[6] G. F. Wredenhagen, "A new method of controller design for systems with input constraints using interpolation functions," in Proc. 33rd Conf. Decision and Control, Lake Buena Vista, FL, 1994, pp. 1024-1029.

[7] G. F. Wredenhagen and P. R. Belanger, "Piecewise-linear LQ control for systems with input constraints," Automatica, vol. 30, pp. 403-416, 1994.

\section{Guaranteed Cost Control of Uncertain Nonlinear Systems Via Polynomial Lyapunov Functions}

Daniel Coutinho, Alexandre Trofino, and Minyue Fu

\begin{abstract}
In this note, we consider the problem of guaranteed cost control for a class of uncertain nonlinear systems. We derive linear matrix inequality conditions for the regional robust stability and performance problems based on Lyapunov functions which are polynomial functions of the state and uncertain parameters. The performance index is calculated over a set of initial conditions. Also, we discuss the synthesis problem for a class of affine control systems. Numerical examples illustrate our method.
\end{abstract}

Index Terms-Convex optimization, guaranteed cost control, uncertain nonlinear systems.

\section{INTRODUCTION}

The development of robustness and performance analysis, as well as design techniques for nonlinear systems, is an important field of research. Despite the existence of powerful techniques to cope with these problems in the context of uncertain linear systems, the generalization to the nonlinear case is a difficult task that has motivated many researchers to study these problems. To deal with nonlinear systems, many control design methods use linear control methodologies applied to quasi-linear parameter varying (LPV) representations [1], or by means of polytopic differential inclusions [2]. For instance, the works of [1] and [3] consider LPV techniques (gain-scheduling), and [4] and [5] use robust controllers. However, these approaches may lead to conservativeness since the nonlinearities of the system are not taken into account and they only consider quadratic Lyapunov functions [6]. Moreover, there are some shortcomings related with the quasi-LPV form that may lead to an infinite-dimensional problem [7] or to the

Manuscript received August 22, 2001; revised January 1, 2002 and March 19, 2002. Recommended by Associate Editor T. Iwasaki. This work was supported in part by CAPES (Brazil) under Grant BEX0784/00-1, by CNPq (Brazil) under Grants 147055/99-7 and 300459/93-9/PQ, and by the Australian Research Council.

D. Coutinho is with the Department of Automation and Systems, Universidade Federal de Santa Catarina, 88040-900 Santa Catarina, Brazil, on leave from the Department of Electrical Engineering, PUC-RS, Porto-Alegre-RS 90619-900, Brazil (e-mail: daniel@ee.pucrs.br).

A. Trofino is with the Department of Automation and Systems, Universidade Federal de Santa Catarina, 88040-900 Santa Catarina, Brazil (e-mail: trofino@das.ufsc.br).

M. Fu is with the School of Electrical Engineering and Computer Science, University of Newcastle, Newcastle, NSW 2308, Australia (e-mail: eemf@ee.newcstle.edu.au).

Publisher Item Identifier 10.1109/TAC.2002.802737. instability of the nonlinear closed-loop system [8]. On the other hand, it is well known that the nonlinear optimal control due to difficulties in the solution of the Hamilton-Jacob equation is not a practical approach [9].

Since the work [10] that showed a solution for rational systems in terms of linear matrix inequalities (LMIs) and based on quadratic Lyapunov functions, some authors have proposed more sophisticated Lyapunov functions to derive less conservative conditions using the LMI framework for uncertain nonlinear systems [11], [6]. The advantage of these techniques over the quasi-LPV or polytopic modeling is that they allow the use of polynomial Lyapunov functions by only requiring that the state and parameter vectors belong to a polytopic set instead of all (state and parameter) nonlinearity. As a result, the number of LMI tests is finite overcoming the problems associated with the quasi-LPV (and/or polytopic) methods for uncertain nonlinear systems.

In this note, we derive LMI conditions for the guaranteed cost control problem for a class of uncertain nonlinear systems. These conditions assure the regional stability of the unforced system and determine a bound on the energy of output signal for a given set of initial conditions. Via an iterative algorithm, this approach is extended to the synthesis problem. The main contributions of this technical note are two fold. First, we consider a polynomial Lyapunov function of the type $v(x, \delta)=x^{\prime} \mathcal{P}(x, \delta) x$, where $\mathcal{P}(x, \delta)$ is a quadratic function of the state $x$ and uncertain parameters $\delta$, that may result in less conservative conditions. Second, the nonlinear system is modeled in an augmented space in which all nonlinearities are taken into account by using scaling matrices associated with them leading to a convex optimization problem in terms of LMI constraints.

The structure of this note is as follows. We state the problem of concern and derive an upper bound on the two-norm of the output performance for a set of initial conditions in Section II. Section III presents an application of the derived method to the guaranteed cost control problem. Section IV presents some concluding remarks.

The notation used in this work is standard. For a real matrix $S, S^{\prime}$ denotes its transpose, $S>0$ means that $S$ is symmetric and positive-definite, and $\mathrm{He}(S)=S+S^{\prime}$. The constant matrices $I_{n}, 0_{n \times m}$ and $0_{n}$ denote $n \times n$ identity matrix, $n \times m$ and $n \times n$ zero matrices respectively. The time derivative of a function $r(t)$ will be denoted by $\dot{r}(t)$ and the argument $(t)$ is often omitted. For two polytopes $\mathcal{B}_{x} \subset \mathbb{R}^{n}$ and $\mathcal{B}_{\delta} \subset \mathbb{R}^{l}$, the notation $\mathcal{B}_{x} \times \mathcal{B}_{\delta}$ represents that $\left(\mathcal{B}_{x} \times \mathcal{B}_{\delta}\right) \subset \mathbb{R}^{(n+l)}$ is a metapolytope obtained by the Cartesian product. The matrix and vector dimensions are omitted whenever they can be determined from the context.

\section{Robustness And Performance of Nonlinear Systems}

Consider the uncertain nonlinear system

$$
\begin{aligned}
\dot{x}=f(x, \delta) & =A(x, \delta) x, \quad x(0)=x_{0} \\
z=h(x, \delta) & =C(x, \delta) x
\end{aligned}
$$

where $x \in \mathbb{R}^{n}$ denotes the state vector, $\delta \in \mathbb{R}^{l}$ denotes the uncertain parameters and $z \in \mathbb{R}^{r}$ denotes the output performance vector.

With respect to the system (1), we consider the following assumptions:

A1) uncertain parameter vector $\delta$, and its time-derivative $\dot{\delta}$ lie in a given polytope $\mathcal{B}_{\delta}$, with known vertices, i.e., $(\delta, \dot{\delta}) \in \mathcal{B}_{\delta}$;

A2) origin $x=0$ of the system is an equilibrium point;

A3) right-hand side of the differential equation is bounded for all values of $x, \delta, \dot{\delta}$ of interest;

A4) $\mathcal{B}_{x}$ is a given polytope specifying a desired neighborhood of the equilibrium point $x=0$ of the system. 